EGU21-12847

https://doi.org/10.5194/egusphere-egu21-12847

EGU General Assembly 2021

(c) Author(s) 2022. This work is distributed under

the Creative Commons Attribution 4.0 License.

\title{
Potential vorticity dynamics of life cycles of blocked weather regimes in the Euro-Atlantic region: A diagnostic framework
}

\author{
Seraphine Hauser ${ }^{1}$, Christian M. Grams ${ }^{1}$, Michael Riemer ${ }^{2}$, Peter Knippertz ${ }^{1}$, and Franziska \\ Teubler $^{2}$ \\ ${ }^{1}$ Karlsruhe Institute of Technology, Institute of Meteorology and Climate Research, Department Troposphere Research, \\ Eggenstein-Leopoldshafen, Germany (seraphine.hauser@kit.edu) \\ ${ }^{2}$ Institute for Atmospheric Physics, Johannes Gutenberg-University Mainz, Mainz, Germany
}

Quasi-stationary, persistent, and recurrent states of the large-scale extratropical circulation, socalled weather regimes, characterize the atmospheric variability on sub-seasonal timescales of several days to a few weeks. Weather regimes featuring a blocking anticyclone are of particular interest due to their long lifetime and potential for high-impact weather. However, state-of-the-art numerical weather prediction and climate models struggle to correctly represent blocking life cycles, which results in large forecast errors at the medium-range to sub-seasonal timescale. Despite progress in recent years, we are still lacking a process-based conceptual understanding of blocked regime dynamics, which hinders a better representation of blocks in numerical models. In particular the relative contributions of dry and moist processes in the onset and maintenance of a block remain unclear.

Here we aim to revisit the dynamics of blocking in the Euro-Atlantic region. To this end we investigate the life cycles of blocked weather regimes from a potential vorticity (PV) perspective in ERA5 reanalysis data (from 1979 to present) from the European Centre for Medium-Range Weather Forecasts. We develop a diagnostic PV framework that allows the tracking of negative PV anomalies associated with blocked weather regimes. Complemented by piecewise PV-tendencies separated into advective and diabatic PV tendencies - we are able to disentangle different physical processes affecting the amplitude evolution of negative PV anomalies associated with blocked regimes. Most importantly, this approach newly enables us to distinguish between the roles of dry and moist dynamics in the initiation and maintenance of blocked weather regimes in a common framework. A first application demonstrates the functionality of the developed PV framework and corroborates the importance of moist-diabatic processes in the initiation and maintenance of a block in a regime life cycle. 